\section{Neurosurgery (Neuro Vascular)}

\section{P.108}

\section{Albumin confers neuroprotection against bilirubin toxicity in experimental intracerebral hemorrhage in mice}

CK Lee (Toronto)* A Alarfaj (Toronto) J Ai (Toronto) B Alharbi (Toronto) $P$ Vasdev (Toronto) RL Macdonald (Toronto)

doi: 10.1017/cjn.2015.216

Background: Blood breakdown products such as bilirubin and bilirubin oxidation products damage cortex and white matter after intracerebral hemorrhage( $\mathrm{ICH})$. Here, we tested whether albumin can antagonize axonal damage caused by bilirubin. Methods: The effect of albumin on white matter injury was investigated using brain slices in vitro. After CD-1 mice brain slices were cut using a vibratome, they were incubated in one of five solutions: artificial cerebral spinal fluid (ACSF), bilirubin ACSF, bilirubin and albumin ACSF, bilirubin ACSF that had albumin added 1 hour(h) later, and bilirubin and denatured albumin ACSF. All solutions were continuously aerated with 95\% O2 and 5\% CO2. Subsequently, electrophysiological recordings of axonal response to electrical stimulation were performed $8 \mathrm{~h}$ after incubation of brain slices. Results: Bilirubin treatment profoundly damaged both myelinated and unmeylinated axons in brain slices, but had a greater effect on myelinated axons. Unmyelinated axons were found to be more susceptible to damage from denatured albumin. Albumin treatment at $0 \mathrm{~h}$ and $1 \mathrm{~h}$ significantly diminished bilirubin toxicity for both myelinated and unmyelinated axons, with $1 \mathrm{~h}$ delayed albumin treatment conferring greater neuroprotection. Conclusions: These results implicate the role of albumin in preventing bilirubin-induced axonal damage following $\mathrm{ICH}$ and its potential therapeutic value for hemorrhagic stroke.

\section{Neurosurgery (Pediatric Neurosurgery)}

\section{P.109}

Impact of preoperative laboratory investigation and blood cross-match on clinical management of pediatric neurosurgical patients

MM Yang (Calgary)* A Singhal (Vancouver) N Au (Vancouver) AR Hengel (Vancouver)

doi: 10.1017/cjn.2015.217

Background: Studies in the literature suggest preoperative laboratory investigations and cross-match are performed unnecessarily and rarely lead to changes in clinical management. This study explored whether preoperative laboratory investigations in neurosurgical children alter clinical management and to determine the utilization of cross-matched blood perioperatively in elective pediatric neurosurgical cases. Methods: We reviewed patient charts for elective neurosurgery procedures (2010-2014) at our institution. Variables collected include preoperative complete blood count (CBC), electrolytes, coagulation, group and screen, and cross-match. Instances of altered clinical management as a consequence of preoperative investigation were noted. The number of cross-matched blood transfused perioperatively was also determined. Results: 477 electively scheduled pediatric neurosurgical patients were reviewed. Preoperative CBC was done on 294 and $39.8 \%$ had at least one laboratory abnormality. Electrolytes and coagulation panels were abnormal in $23.8 \%$ and $24.5 \%$ respectively. The preoperative investigations led to a change in clinical management in three patients, two of which were associated with significant past medical history. $57.9 \%$ had blood cross-matched and $3.6 \%$ of patients received perioperative blood transfusions. The cross-match to transfusion ratio was 16. Conclusion: This study suggests that the results of preoperative laboratory exams have limited value, apart from cases with oncology and complex pre-existing conditions. Additionally, cross-matching might be excessively conducted in elective pediatric neurosurgical cases.

\section{Neurosurgery (SPINE)}

\section{P.110}

The association of cervical spine alignment with neurological recovery in a prospective cohort of surgical myelopathy patients: analysis of a series of 124 cases

MF Shamji (Toronto)* C Mohanty (Toronto) EM Massicotte (Toronto) $M G$ Fehlings (Toronto)

doi: 10.1017/cjn.2015.218

Objective: The impact of spinal alignment on neurological recovery among myelopathy patients has not been thoroughly investigated. This study evaluated the impact of sagittal cervical alignment on neurological recovery in a prospective surgical series of myelopathy patients. Methods: Prospective data was analyzed from surgical CSM patients at a tertiary-care neurosurgical centre. Demographic data and clinical preoperative and postoperative measures of neurological disability (mJOA, Nurick, NDI scores) were analyzed for dependency on cervical spine imaging parameters. Results: Among 124 CSM patients, 34\% exhibited kyphotic alignment. Surgical intervention was more frequently anterior or combined anterior/posterior among this group than those with preserved lordosis. Most patients exhibited postoperative neurological improvement for myelopathy severity, however the extent of this improvement was dichotomous based on preoperative sagittal alignment. Improvement was greater among patients with preoperative lordosis $(\triangle \mathrm{mJOA}$ of 3.1$)$ than those with preoperative kyphosis $(\Delta \mathrm{mJOA}$ of $1.4, \mathrm{p}=0.02)$. Surgical correction of spinal malalignment did not provide for heightened neurological recovery, although whether it protects against symptomatic adjacent segment disease is unclear. Conclusion: Most CSM patients showed postoperative neurological improvement. Patients with preoperative lordotic alignment exhibited greater improvement than those with preoperative kyphotic alignment. Neither correction of the spinal alignment nor surgical approach in this series specifically affected the extent of neurological recovery. 